\title{
Exosomal miR-30d-5p of neutrophils induces M1 macrophage polarization and primes macrophage pyroptosis in sepsis-related acute lung injury
}

Yang Jiao ${ }^{1,2}$, Ti Zhang $^{3}$, Chengmi Zhang ${ }^{2}$, Haiying $\mathrm{Ji}^{2}$, Xingyu Tong ${ }^{2}$, Ran Xia ${ }^{2}$, Wei Wang ${ }^{2}$, Zhengliang Ma ${ }^{{ }^{*}}$ and Xueyin $\mathrm{Shi}^{2^{*}}$ (D)

\begin{abstract}
Background: Polymorphonuclear neutrophils (PMNs) play an important role in sepsis-related acute lung injury (ALI). Accumulating evidence suggests PMN-derived exosomes as a new subcellular entity acting as a fundamental link between PMN-driven inflammation and tissue damage. However, the role of PMN-derived exosomes in sepsis-related $\mathrm{ALI}$ and the underlying mechanisms remains unclear.

Methods: Tumor necrosis factor-a (TNF-a), a key regulator of innate immunity in sepsis-related ALI, was used to stimulate PMNs from healthy C57BL/6J mice in vitro. Exosomes isolated from the supernatant were injected to C57BL/6J wild-type mice intraperitoneally (i.p.) and then examined for lung inflammation, macrophage $(\mathrm{M} \varphi$ ) polarization and pyroptosis. In vitro co-culture system was applied where the mouse Raw264.7 macrophages or bone marrow-derived macrophages (BMDMs) were co-cultured with PMN-derived exosomes to further confirm the results of in vivo animal study and explore the potential mechanisms involved.

Results: Exosomes released by TNF-a-stimulated PMNs (TNF-Exo) promoted M1 macrophage activation after in vivo i.p. injection or in vitro co-culture. In addition, TNF-Exo primed macrophage for pyroptosis by upregulating NOD-like receptor 3 (NLRP3) inflammasome expression through nuclear factor KB (NF-KB) signaling pathway. Mechanistic studies demonstrated that miR-30d-5p mediated the function of TNF-Exo by targeting suppressor of cytokine signaling (SOCS-1) and sirtuin 1 (SIRT1) in macrophages. Furthermore, intravenous administration of miR-30d-5p inhibitors significantly decreased TNF-Exo or cecal ligation and puncture (CLP)-induced M1 macrophage activation and macrophage death in the lung, as well as the histological lesions.
\end{abstract}

Conclusions: The present study demonstrated that exosomal miR-30d-5p from PMNs contributed to sepsisrelated ALI by inducing M1 macrophage polarization and priming macrophage pyroptosis through activating NF-KB

\footnotetext{
*Correspondence: mazhengliang1964@nju.edu.cn; shixueyin1128@163.com

${ }^{1}$ Department of Anesthesiology, Nanjing Drum Tower Hospital, The

Affiliated Hospital of Nanjing University Medical School, 321 Zhongshan

Road, Nanjing 210008, China

2 Department of Anesthesiology and Intensive Care Unit, Xinhua Hospital,

School of Medicine, Shanghai Jiaotong University, 1665 Kongjiang Road,

Shanghai 200092, China

Full list of author information is available at the end of the article
}

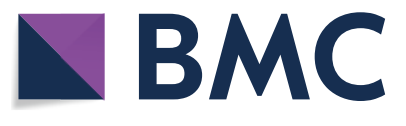

(c) The Author(s) 2021. Open Access This article is licensed under a Creative Commons Attribution 4.0 International License, which permits use, sharing, adaptation, distribution and reproduction in any medium or format, as long as you give appropriate credit to the original author(s) and the source, provide a link to the Creative Commons licence, and indicate if changes were made. The images or other third party material in this article are included in the article's Creative Commons licence, unless indicated otherwise in a credit line to the material. If material is not included in the article's Creative Commons licence and your intended use is not permitted by statutory regulation or exceeds the permitted use, you will need to obtain permission directly from the copyright holder. To view a copy of this licence, visit http://creativecommons.org/licenses/by/4.0/. The Creative Commons Public Domain Dedication waiver (http://creativeco mmons.org/publicdomain/zero/1.0/) applies to the data made available in this article, unless otherwise stated in a credit line to the data. 
signaling. These findings suggest a novel mechanism of PMN-M $\varphi$ interaction in sepsis-related ALI, which may provide new therapeutic strategies in sepsis patients.

Keywords: Sepsis-related acute lung injury, Neutrophil, Macrophage, Exosomes, miR-30d-5p, Pyroptosis

\section{Introduction}

Sepsis is defined as a global health priority by the World Health Organization (WHO) and characterized by excessive inflammation in response to infection, with a reported death rate of $30-45 \%$ in hospitalized patients $[1,2]$. Acute respiratory distress syndrome (ARDS) is the most common severe manifestation of multiple organ dysfunction syndrome and a significant factor contributing to the morbidity and mortality of sepsis [3].

Polymorphonuclear neutrophils (PMNs) are the most abundant leukocytes in mammals, which play a crucial role in the pathogenesis of sepsis-related acute lung injury (ALI) or ARDS [4]. Exosomes are small extracellular vesicles secreted by various cell types, with size ranging from 30 to $150 \mathrm{~nm}$ [5]. They can transfer a multitude of proteins and genetic material (including DNA, mRNA and microRNA [miRNA]) to target cells, playing a key role in cell-to-cell communications [6]. Animal studies have implicated the roles of PMN-derived exosomes in many chronic lung injuries, including chronic obstructive pulmonary disease (COPD), bronchopulmonary dysplasia and asthma [7, 8], but their role in sepsis-related ALI remains unclear.

In addition to PMNs, M1 macrophages, as a proinflammatory phenotype, also promoted the occurrence of sepsis-related ALI. M1 macrophages could be activated by circulating plasma exosomes, which further promote the secretion of proinflammatory cytokines, such as interleukin $1 \beta$ (IL-1 $\beta$ ), IL-12, IL- 6 and tumor necrosis factor- $\alpha$ $(\mathrm{TNF}-\alpha)[9,10]$. Recently, the pyroptosis of macrophage $(\mathrm{M} \phi)$ has also been highlighted in sepsis-related ALI. Pyroptosis is a caspase-1-dependent proinflammatory cell death type $[4,11]$. Caspase- 1 activated by inflammasomes including NOD-like receptor 3 (NLRP3) inflammasomes initiates pyroptosis by cleaving gasdermin $\mathrm{D}$ (GSDMD, the core event in pyroptosis) to form pores in the plasma membrane, leading to cell swelling and membrane rupture, finally resulting in the leakage of mature forms of IL- $1 \beta$ and IL-18 out of cells $[12,13]$. Pyroptotic macrophage-released danger signals or danger-associated molecular pattern molecules enhance inflammatory responses in sepsis-related ALI [14].

The crosstalk between PMNs and macrophages in regulating inflammation has been documented [15-18]. We previously reported that exosomes secreted from macrophages promoted neutrophil necroptosis following hemorrhagic shock [17]. Macrophage pyroptosis in sepsis could also be primed by neutrophil extracellular traps (NETs) [19]. However, the effects of PMN-derived exosomes on the behavior of macrophage and the underlying mechanisms in sepsis-related ALI are unknown.

Here, we firstly identified the role of PMN-derived exosomes in sepsis-related ALI by inducing macrophage M1 polarization and priming macrophage for pyroptosis. Bioinformatics analysis and further mechanistic studies revealed that PMN-derived exosomes transferred miR-30d-5p into macrophages and then activated NF- $\mathrm{kB}$ signaling pathway by inhibiting SOCS-1 (suppressor of cytokine signaling) and sirtuin 1 (SIRT1), which were both recognized as negative regulators of NF- $\mathrm{KB}$ signaling pathway previously $[20,21]$. These findings suggest a previously unidentified pathway of $\mathrm{PMN}-\mathrm{M} \phi$ crosstalk, which could enhance macrophage activation and death, and subsequently exaggerate post-sepsis inflammation and induce lung injury.

\section{Materials and methods \\ Animals}

Wild type (WT) male C57BL/6J mice aged 6-8 weeks (Shanghai Sippr-BK Laboratory Animal Co., Ltd., Shanghai, China) were fed under a specific pathogen-free environment in Xinhua Hospital Animal Laboratory (Shanghai, China). All animal experiments were conducted under the rules approved by the Ethics Committee of Xinhua Hospital Affiliated to Shanghai Jiao Tong University School of Medicine (Approval No.: XHEC-F-2020-019).

\section{PMNs isolation and activation}

PMNs were induced in the peritoneal cavity of the mice as previously described [22]. Briefly, mice were injected intraperitoneally (i.p.) with $1 \mathrm{ml} 9 \%$ casein solution twice overnight and killed $3 \mathrm{~h}$ after the second injection to harvest the peritoneal lavage fluid (PLF), which was subsequently centrifuged, and the cell pellets were washed. PMNs were isolated by discontinuous density gradient centrifugation with two commercially available solutions (Histopaque-1077 and Histopaque-1119) of differential density (Sigma (St. Louis, MO; \#11191 and \#10771) according to the manufacturer's instructions. The resulting cells consisted of $90 \%$ PMNs, and viability of the isolated PMNs was $95 \%$ as assessed by flow cytometry and Trypan blue staining, respectively. 
After isolation, PMNs were suspended in complete culture medium (RPMI 1640 containing 10\% exosome-free FBS, supplemented with $50 \mathrm{mg} / \mathrm{ml}$ penicillin/streptomycin) at a concentration of $10^{6}$ cells $/ \mathrm{ml}$. PMNs activation was induced upon 12-h incubation with $20 \mathrm{ng} / \mathrm{mL}$ TNF- $\alpha$ at $37{ }^{\circ} \mathrm{C}$. An equal volume of phosphate buffered saline (PBS) to TNF- $\alpha$ was used as negative control.

\section{Exosome isolation and characterization}

Exosomes were isolated from the supernatant of PMNs treated with PBS (PBS-Exo) or TNF- $\alpha$ (TNF-Exo) ex vivo using Total Exosome Isolation Reagent (\#4484450; Thermo Fisher Scientific, Waltham, MA, USA). The detailed isolation procedure and the methods used to determine exosomal morphology, size distribution, and surface marker expression are described in Additional file 1.

\section{In vivo exosome administration to $\mathrm{WT}$ C57BL/6 mice}

To explore exosome function in vivo, five WT C57BL/6 mice in each group were injected with PBS-Exo or TNFExo $(300 \mu \mathrm{g} /$ mouse $)$ intraperitoneally using a 31 -gauge insulin syringe, respectively. An equal volume of PBS was used as negative control. After $24 \mathrm{~h}$, the obtained peritoneal lavage fluid was centrifuged, and peritoneal macrophages were detected by flow cytometry after gating with F4/80. To visualize changes in morphology and macrophage polarization, lung tissues were harvested and fixed in $4 \%$ paraformaldehyde for H\&E and immunofluorescence staining. H\&E staining was evaluated by a pathologist who was blinded to the experimental groups. To evaluate the lung injury, five independent random lung fields were evaluated per mouse for neutrophils in alveolar spaces, neutrophils in interstitial spaces, hyaline membranes, proteinaceous debris filling the airspaces, and alveolar septal thickening, and weighed according to the official American Thoracic Society workshop report on features and measurements of experimental ALI in animals [23]. The resulting injury score is a continuous value between 0 and 1 . For immunofluorescence staining, paraffin-embedded lung tissues were sectioned, blocked with PBS containing $1 \%$ goat serum and $3 \%$ BSA, permeabilized with PBS/Triton $0.01 \%$, and incubated with F4/80 and iNOS antibodies, and then with species-specific secondary antibodies coupled with Alexa Fluor Dyes. DNA was stained using DAPI. The sections were treated with autofluorescent quenching solution (\#G1221; Servicebio, Wuhan, China) and mounted in Vectashield Mounting Media.

The in vivo miR-30d-5p inhibitors were transfected into the mouse lung through tail vein injection using the in vivo-jetPEI (Polyplus-transfection SA, New York, NY). Briefly, the miR-30d-5p inhibitor or negative control
(50 $\mu \mathrm{g}, \mathrm{N} / \mathrm{P}$ ratio $=6$, i.e., $0.12 \mu \mathrm{l}$ of in vivo-jetPEI per $\mu \mathrm{g}$ nucleic acid) dissolved in $200 \mu \mathrm{l} 5 \%$ glucose solution was injected into each mouse 1 day before exosome injection, according to the manufacturer's protocol.

\section{In vitro co-culture experiments}

Raw264.7 macrophages or BMDMs were treated with PMN-derived exosomes $(100 \mu \mathrm{g} / \mathrm{ml})$ at $37{ }^{\circ} \mathrm{C}$ for $24 \mathrm{~h}$. To induce pyroptosis, macrophages were primed with exosomes for $24 \mathrm{~h}$ before stimulation with $5 \mathrm{mM}$ ATP (\#HY-B2176; MedChemExpress, Monmouth Junction, NJ, USA) or $20 \mu \mathrm{M}$ nigericin (\#HY-100381; MedChemExpress) for $2 \mathrm{~h}$.

For miR-30d-5p inhibition, Raw264.7 macrophages were transfected with microRNA control or miR-30d-5p inhibitor (Guangzhou Ribobio Corporation, Guangzhou, China) at a concentration of $50 \mathrm{nM}$ using Lipofectamine 3000 for $24 \mathrm{~h}$ prior to be co-cultured with exosomes as per the manufacturer's instructions. For miR-30d-5p overexpression, Raw264.7 macrophages were transfected with microRNA control or miR-30d-5p mimic at a concentration of $50 \mathrm{nM}$ using Lipofectamine 3000 for $48 \mathrm{~h}$.

\section{Flow cytometry analysis of M1 polarization and pyroptosis} Macrophages were centrifuged and resuspended in PBS for FACS analysis. According to the manufacturer's instructions, anti-CD11c (\#117307; BioLegend, San Diego, CA, USA), anti-CD86 (\#105106; BioLegend), antiCD206 (\#141706; BioLegend) and anti-F4/80 (\#123116; BioLegend) antibodies were used for fluorescent staining. Isotype antibody controls were used to exclude nonspecific staining. Data were obtained using a CytoFLEX flow cytometer (Beckman, USA). Programmed cell death was analyzed with apoptosis detection kit (\#559763; BD Biosciences, Franklin Lakes, NJ). Macrophages were incubated with Annexin-V and PI for $15 \mathrm{~min}$ at room temperature in the dark and then analyzed by flow cytometry. Cells double-stained positive for Annexin V and PI were considered as undergoing programmed death. Cell pyroptosis was detected by twocolor flow cytometry. Macrophages were incubated with Alexa Fluor 488-labeled caspase-1 FLICA (\#ICT098; ImmunoChemistry, Bloomington, MN, USA) at $37{ }^{\circ} \mathrm{C}$ for $1 \mathrm{~h}$. After being fixed with 4\% paraformaldehyde, cells were stained with TMR red-labeled In-Situ Cell Death Detection reagent (\#12156792910; Roche Applied Science, Indianapolis, IN, USA), following the manufacturer's instructions. Double-stained cells were identified as pyroptotic cells. Background and autofluorescence were determined by a control antibody with the same isotype staining. 
Sequencing of exosomal miRNA and data analysis

Total RNA was extracted from PBS-Exo/TNF-Exo using the miRNeasy Serum/Plasma Kit (Qiagen, Valencia, CA, USA). The final ligation PCR products were sequenced using the BGISEQ-500 platform (BGI Group, Shenzhen, China). After acquiring the raw data, the differentially expressed miRNAs were calculated using the $t$ test. Those with $\geq$ twofold upregulation and a $P$ value $<0.05$ were regarded as significantly different. A heat map was generated using the R 3.5.3 software. Pathway analysis was conducted using the Kyoto Encyclopedia of Genes and Genomes (KEGG) pathway database. The 20 most enriched pathways related to signaling transduction are listed and were used to reveal the most associated pathways.

\section{Quantitative real-time PCR (RT-qPCR)}

Total RNA isolation was performed using TRIzol following the manufacturer's instruction (TAKARA, Japan). mRNA was reverse transcribed using PrimeScript RT reagent Kit (\#RR036; TAKARA, Tokyo, Japan), and PCR was conducted using TB Green ${ }^{\mathrm{TM}}$ Premix Ex Taq ${ }^{\mathrm{TM}}$ (Tli RNaseH Plus) (\#RR420A; TAKARA) and QuantStudio ${ }^{\text {TM }}$ 3 System (Applied Biosystems). Data were normalized to the expression of GAPDH. Primer sequences are shown in Additional file 1: Table S1.

For exosomal miRNA quantification, total RNA was extracted from $200 \mu \mathrm{l}$ PMN-derived exosomes using miRNeasy Serum/Plasma kit (\#217184; Qiagen, Valencia, CA, USA). RNA pellets were resuspended in $14 \mu \mathrm{l}$ RNasefree water. Twelve microliters of RNA solution were used for reverse transcription, according to the protocol of miScript RT Kit (\#218161; Qiagen). miRNA expression was quantified using a miScript SYBR Green PCR Kit (\#218075; Qiagen). qPCR analysis was also performed for miR-30d-5p expression in cells or the lung tissue. Briefly, RNA was extracted by TRIzol reagent (TAKARA, Japan) and RT-qPCR was conducted using the Mir-X miRNA qRT-PCR SYBR Kit (\#638316; TAKARA). Relative expression was calculated using the comparative cycle threshold $(\mathrm{Ct})$ method $\left(2^{-\Delta \Delta \mathrm{CT}}\right)$ normalized to U6. The miRNA qPCR primers were purchased from Guangzhou Ribobio Corporation.

\section{Luciferase assay}

The 3 '-UTR of the SOCS-1/SIRT1 sequence containing the predicted miR-30d-5p binding sites and its mutant was cloned into the plasmid vector and transfected into HEK293 cells. A renilla luciferase vector was co-transfected in all transfections described to monitor transfection efficiency. All luciferase results were reported as relative light units: the average of the photinus pyralis firefly activity observed was divided by the average of the activity recorded from the renilla luciferase vector.

\section{Mouse model of cecal ligation and puncture (CLP)}

The CLP mouse model was prepared as previously described [22]. Mice were anesthetized with ketamine $(50 \mathrm{mg} / \mathrm{kg})$ and xylazine $(5 \mathrm{mg} / \mathrm{kg})$ via i.p. injection. After disinfection, a $1 \mathrm{~cm}$ midline laparotomy was made in the abdomen. The cecum was then exteriorized, and ligated below the cecal valve, and punctured with an 18-gauge needle to induce sepsis. A small drop of cecal content was extruded. The cecum was then returned to the peritoneal cavity and the abdominal incision closed with sutures. Mice were resuscitated with $(5 \mathrm{ml} / 100 \mathrm{~g})$ saline. Sham animals underwent the same surgical procedures without cecum ligation and puncture. The in vivo miR-30d-5p inhibitors were transfected into the mouse lung through tail vein injection using the in vivo-jetPEI one day before CLP surgery, according to the manufacturer's protocol. At $24 \mathrm{~h}$ after surgery, the animals were euthanized with phenobarbital overdose $(100 \mathrm{mg} / \mathrm{kg}$ body weight), followed by collection of the lung tissues as described previously.

\section{Statistics}

The normal distribution of the data was tested using the Shapiro-Wilk test. Normally distributed data are presented as means \pm SEM. Comparisons between 2 groups were performed by the 2-tailed Student's t test. Multiple group comparisons were performed by one-way ANOVA followed by Tukey's multiple comparisons test with GraphPad Prism 8 software. Comparison of survival rates between groups was performed using Log-rank test. A value of $P<0.05$ was considered statistically significant.

\section{Results}

TNF-Exo induces lung injury by affecting M1 macrophage activation and pyroptosis in vivo

TNF- $\alpha$ is a potent inducer of inflammatory response and a key regulator of innate immunity in sepsis-related ALI, which is often used to activate neutrophils [24-26]. Thus, PMNs from healthy C57BL/6J mice were treated with PBS (PBS-Exo) or stimulated with TNF- $\alpha$ (TNFExo) ex vivo to isolate exosomes. Morphologically, transmission electron microscopy (TEM) showed that these isolated microvesicles displayed a round, cup-shaped morphology (Additional file 2: Fig. S1A), with a diameter about $70 \mathrm{~nm}$ (Additional file 2: Fig. S1B). Western blot further confirmed high expression of exosome-specific markers CD9, CD63 and TSG101 (Additional file 2: Fig. $\mathrm{S} 1 \mathrm{C})$. 
TNF-Exo was injected i.p. to C57BL/6J WT mice to determine whether TNF-Exo could induce lung inflammation. Fluorescence imaging showed that TNF-Exo accumulated in the lung tissue after TNF-Exo injection (Fig. 1a). Histological lesions were observed in the mouse lung after the administration of TNF-Exo compared with PBS-Exo (Fig. 1b). Meanwhile, the proinflammatory mediators (iNOS, IL- $1 \beta$ and TNF- $\alpha$ ) in the lung tissue were highly expressed and the number of local M1 macrophages (iNOS + F4/80 + cells) was significantly increased (Fig. 1c, d), suggesting that the lung inflammation induced by TNF-Exo was in parallel with the macrophage inflammatory activity. Furthermore, we found that TNF-Exo significantly increased the proportion of M1 macrophage (CD11c + CD206-) and dying cells $($ Annexin $\mathrm{V}+\mathrm{PI}+$ ) in peritoneal macrophage $(\mathrm{PM} \phi)$

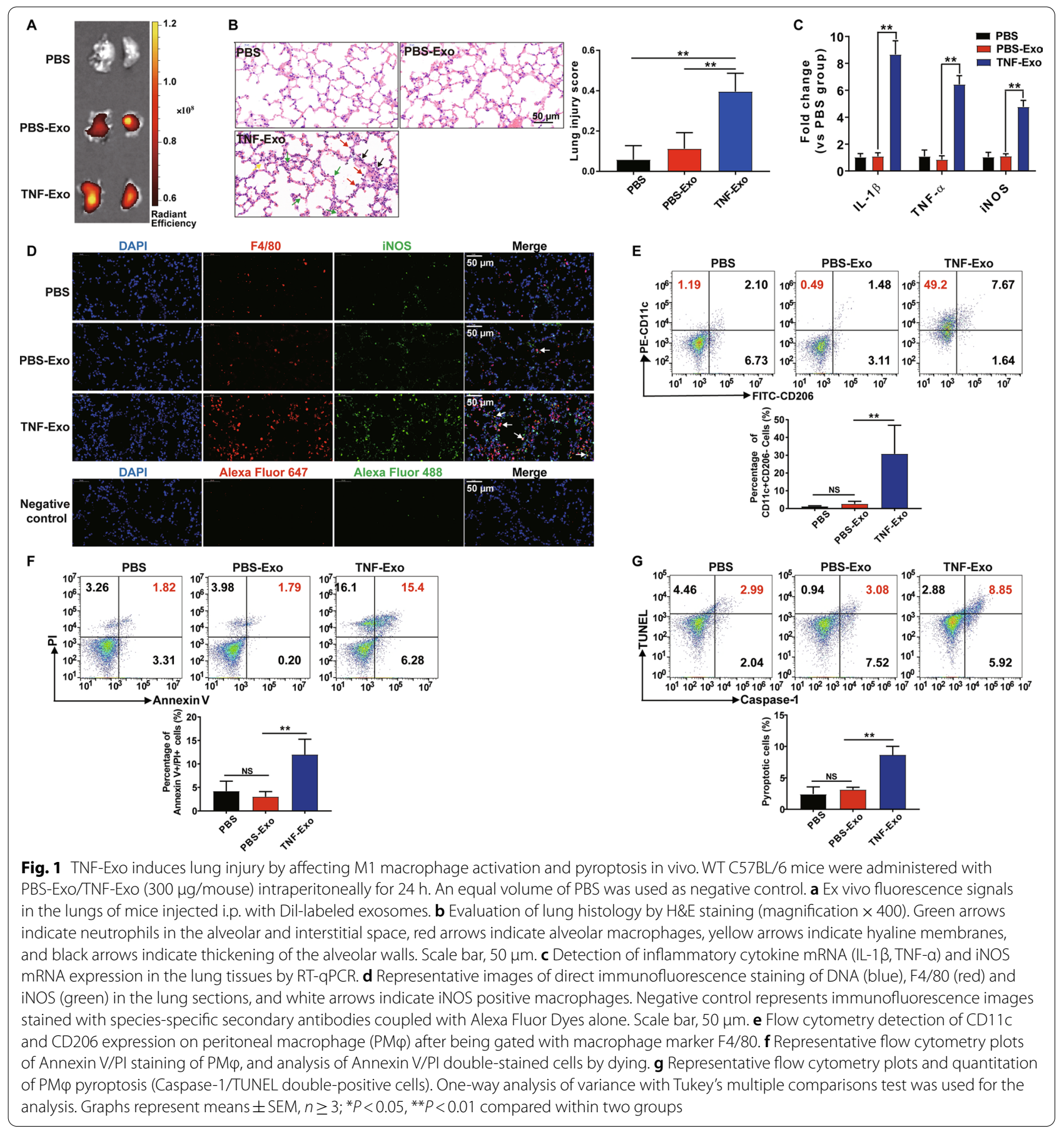


as assessed by flow cytometry (Fig. 1e, f). To further determine the type of $\mathrm{PM} \phi$ death, $\mathrm{PM} \phi$ was detected for nuclear fragmentation, caspase- 1 activation (the characteristics of pyroptosis) by flow cytometry and the result showed $\sim 8$ pyroptotic $\mathrm{PM} \phi$ at $24 \mathrm{~h}$ after TNF-Exo injection (Fig. 1g). All these data indicate that TNF-Exo in vivo could induce pulmonary inflammation potentially due to M1 macrophage activation and pyroptosis.
TNF-Exo promotes M1 macrophage activation and primes macrophage pyroptosis through NF-KB pathway in an in vitro co-culture model

Using an in vitro co-culture system, macrophages were co-cultured with PMN-derived exosomes to further confirm the results of the in vivo animal study. First, we observed the direct transfer of exosomes between PMNs and $M \phi$, as evidenced by $\mathrm{M} \phi$ internalization of PMN-derived exosomes using confocal microscopy (Additional file 2: Fig. S2A). In addition, no matter in primary cell or cell line of macrophage, TNF-Exo could promote macrophage M1 polarization (Fig. 2a-c, Additional file 2: S2B-S2D). However, TNF-Exo did not

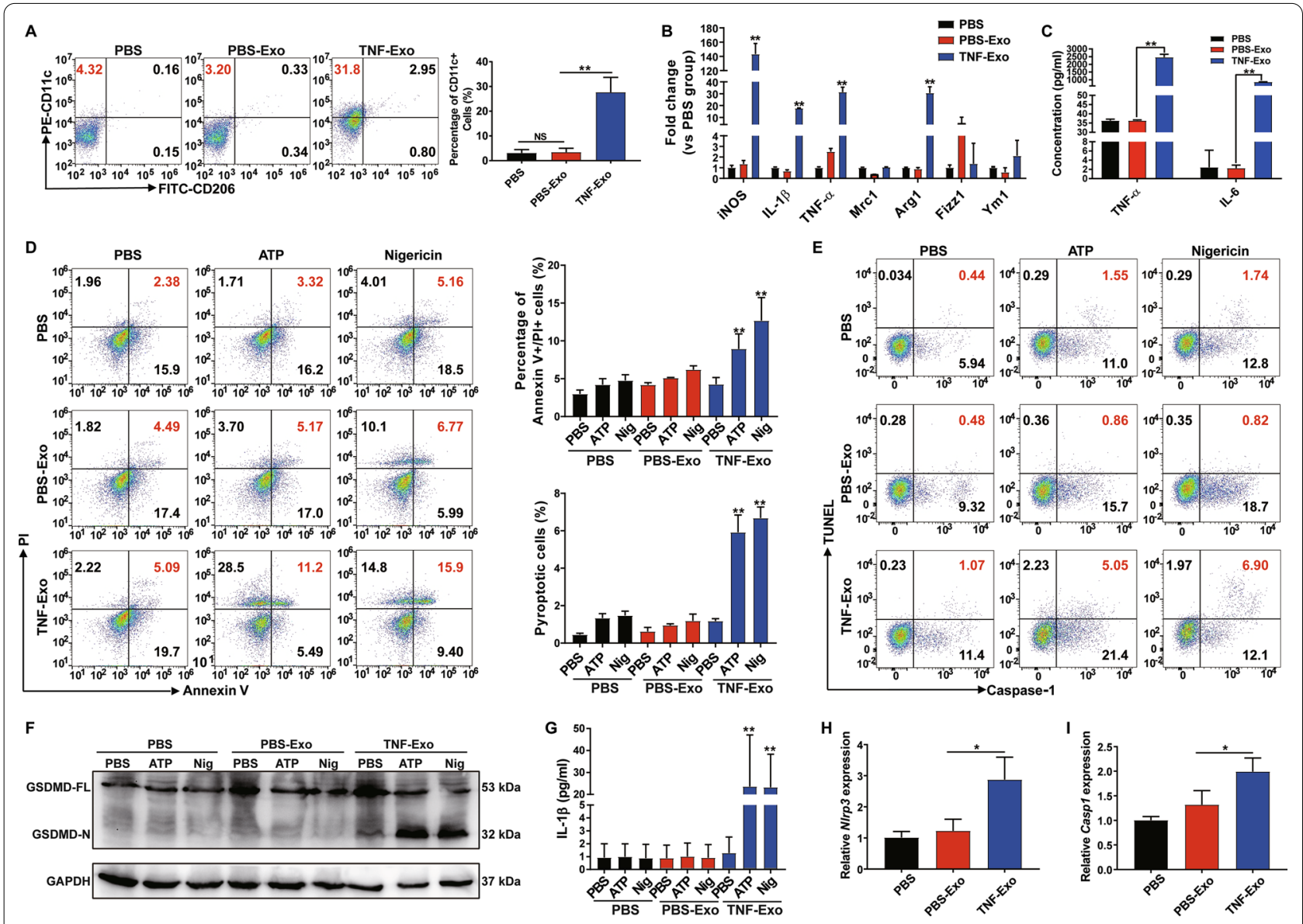

Fig. 2 TNF-Exo promotes M1 macrophage activation and primes macrophage pyroptosis through NF-KB pathway in an in vitro co-culture model. a-c Treatment of Raw264.7 macrophages with PBS-Exo/TNF-Exo for $24 \mathrm{~h}$. An equal volume of PBS was used as negative control. a Flow cytometry detection of CD11 c and CD206 expression on Raw264.7 macrophages. b Detection of expression levels of iNOS, IL-1 $\beta$, TNF-a, Mrc1, Arg1, Fizz1 and Ym1 mRNA by RT-qPCR. c Detection of the concentration of inflammatory cytokines (IL-6, TNF-a) in the supernatant of Raw264.7 macrophages by ELISA. d-g Stimulation of Raw264.7 macrophages with PBS/PBS-ExO/TNF-Exo for $24 \mathrm{~h}$ and treatment with PBS, $5 \mathrm{mM}$ ATP or $20 \mathrm{mM}$ nigericin for 2 h. $\mathbf{d}$ Flow cytometry evaluation of macrophage death by Annexin-V and PI double-staining. e Flow cytometry evaluation of macrophage pyroptosis by Caspase-1 and TUNEL double-staining. $\mathbf{f}$ Cleavage of GSDMD by western blot. GSDMD-FL: full-length GSDMD, GSDMD-N: N-terminal cleavage products of GSDMD. $\mathbf{g}$ Analysis of culture supernatants for IL-1 $\beta$ secretion by ELISA. $\mathbf{h}$-i Treatment of Raw264.7 macrophages with PBS/ PBS-EXo/TNF-Exo for $24 \mathrm{~h}$, and detection of NLRP3 and caspase-1 mRNA expression by RT-qPCR. One-way analysis of variance with Tukey's multiple comparisons test was used for the analysis. Graphs represent means $\pm S E M, n \geq 3 ;{ }^{*} P<0.05,{ }^{* *} P<0.01$ compared within two groups 
promote macrophage death or pyroptosis directly; after adding ATP/nigericin, pyroptotic cell death of TNFExo-primed macrophages was significantly upregulated (Fig. 2d-e). ATP/nigericin is often used as positive controls for NLRP3 inflammasome activation by initiating the assembly of inflammasomes [27]. Activated NLRP3 inflammasomes splice IL-1 $\beta$ precursor for maturation and secretion, and cleave GSDMD to initiate pyroptosis. The cleaved GSDMD N-terminus and IL- $1 \beta$ secretion were increased in TNF-Exo plus ATP or nigericin group as assessed by Western blot and ELISA, respectively (Fig. 2f-g). Besides, TNF-Exo increased NLRP3 and caspase-1 mRNA expressions in macrophages as measured by RT-qPCR (Fig. $2 \mathrm{~h}-\mathrm{i}$ ). These data suggest that TNFExo only served as a priming signal to upregulate NLRP3 inflammasome expression, while the second signals, such as ATP or nigericin, were required to finally induce macrophage pyroptosis in vitro.

Next, we wanted to determine through which pathway TNF-Exo promoted M1 macrophage activation and primed macrophage pyroptosis in vitro by co-culturing macrophages with exosomes by proteomics (Additional file 3). The proteomic results were consistent with the above finding that TNF-Exo significantly upregulated the expression of M1 macrophage marker (inducible nitric oxide synthase, iNOS) and the expression of NLRP3 complexes (NLRP3 and Caspase-1) (Additional file 2: S2E). Enrichment pathway analysis showed that NF- $\mathrm{kB}$ signaling pathway was within the 20 most enriched pathways (Additional file 2: S2F), and Western blot demonstrated that NF- $\mathrm{kB}$ activity was increased in TNF-Exo-treated macrophages (Additional file 2: S2G). The proinflammatory transcription factor NF- $\mathrm{kB}$ is known to regulate M1 macrophage polarization and NLRP3 inflammasome expression [20,28]. Taken together, the above results suggested that TNF-Exo upregulated NF- $\mathrm{kB}$ signaling activity to promote $\mathrm{M} 1$ macrophage activation and prime macrophage for pyroptosis.

\section{miRNA analysis of PMN-derived exosomes}

Knowing that exosomes from septic shock patients can convey miRNAs related to pathogenic pathways, which may represent a novel mechanism for intercellular communication during sepsis [29], we subsequently screened PMN-derived exosomes for miRNAs and detected 26 miRNAs. It was found that they increased by $\geq$ twofold in TNF-Exo as compared those in PBS-Exo (Fig. 3a, Additional file 2: Table S1). Enrichment pathway analysis was also performed to identify the most enriched pathways related to signaling transduction for these 26 miRNAs, and the data showed that NF- $\mathrm{kB}$ signaling pathway was within the 20 most enriched pathways (Fig. 3b).
Next, we searched the literature and found that miR30d-5p expression was positively correlated with NF- $\mathrm{kB}$ signaling pathway [30]. We thus used RT-qPCR and found that the expression of miR-30d-5p in TNF-Exo was significantly higher than that in PBS-Exo (Fig. 3c). It was surprisingly found that the level of miR-30d-5p in PMNs was decreased after TNF- $\alpha$ stimulation, indicating that miR-30d-5p relocated from the cellular compartment to exosomes (Fig. 3d). Interestingly, macrophages exhibited a higher level of miR-30d-5p after being cultured with TNF-Exo in vitro (Fig. 3e). These results indicate that TNF- $\alpha$ could enhance miR-30d-5p loading into exosomes from PMNs and transferring to recipient macrophages. Thus, we hypothesized that TNF-Exo may transfer miR$30 \mathrm{~d}-5 \mathrm{p}$ into macrophages and then activate NF- $\mathrm{kB}$ signaling pathway.

\section{TNF-Exo activates NF-KB signaling pathway in macrophage via miR-30d-5p}

To test the above hypothesis, we transfected Raw264.7 macrophages with miR-30d-5p inhibitors prior to culturing with TNF-Exo. It was found that transfection of miR-30d-5p inhibitors reversed the upregulation of M1 macrophage markers and pro-inflammatory cytokines induced by TNF-Exo (Fig. $4 \mathrm{a}-\mathrm{c}$ ). In addition, inhibition of miR-30d-5p significantly decreased NF- $\mathrm{kB}$ p-p65 protein expression in recipient macrophages treated with TNF-Exo (Fig. 4d).

Moreover, treatment of Raw264.7 macrophages with a miR-30d-5p inhibitor prior to culturing with TNFExo decreased the mRNA level of NLRP3 and caspase-1 (Fig. 4e). Intracellular caspase-1 activation was also measured by flow cytometry and Western blot. Transfection of miR-30d-5p inhibitors exhibited a significant suppressive effect on caspase-1 activation in response to TNF-Exo plus ATP (Fig. 4f-g). The cleaved GSDMD N-terminus upregulated by TNF-Exo plus ATP stimulation was also inhibited by miR-30d-5p inhibitors (Fig. 4h). These data show that exosomal miR-30d-5p promoted M1 macrophage activation and primed macrophage for pyroptosis through activating NF- $\mathrm{kB}$ signaling pathway.

\section{Exosomal miR-30d-5p activates NF-KB in macrophage via targeting SOCS-1 and SIRT1}

Next, we sought to understand the mechanism through which miR-30d-5p activated NF- $\mathrm{kB}$ signaling pathway. Bioinformatics analysis showed that SOCS-1 (suppressor of cytokine signaling) and sirtuin 1 (SIRT1) were putative target genes for miR-30d-5p, and also negative regulators of NF- $\kappa B$ signaling pathway. As predicted by Targetscan in our study, miR-30d-5p may conserve the binding sites in the 3' UTR of SOCS-1 and SIRT1 (Fig. 5a). To validate this bioinformatic prediction, we 

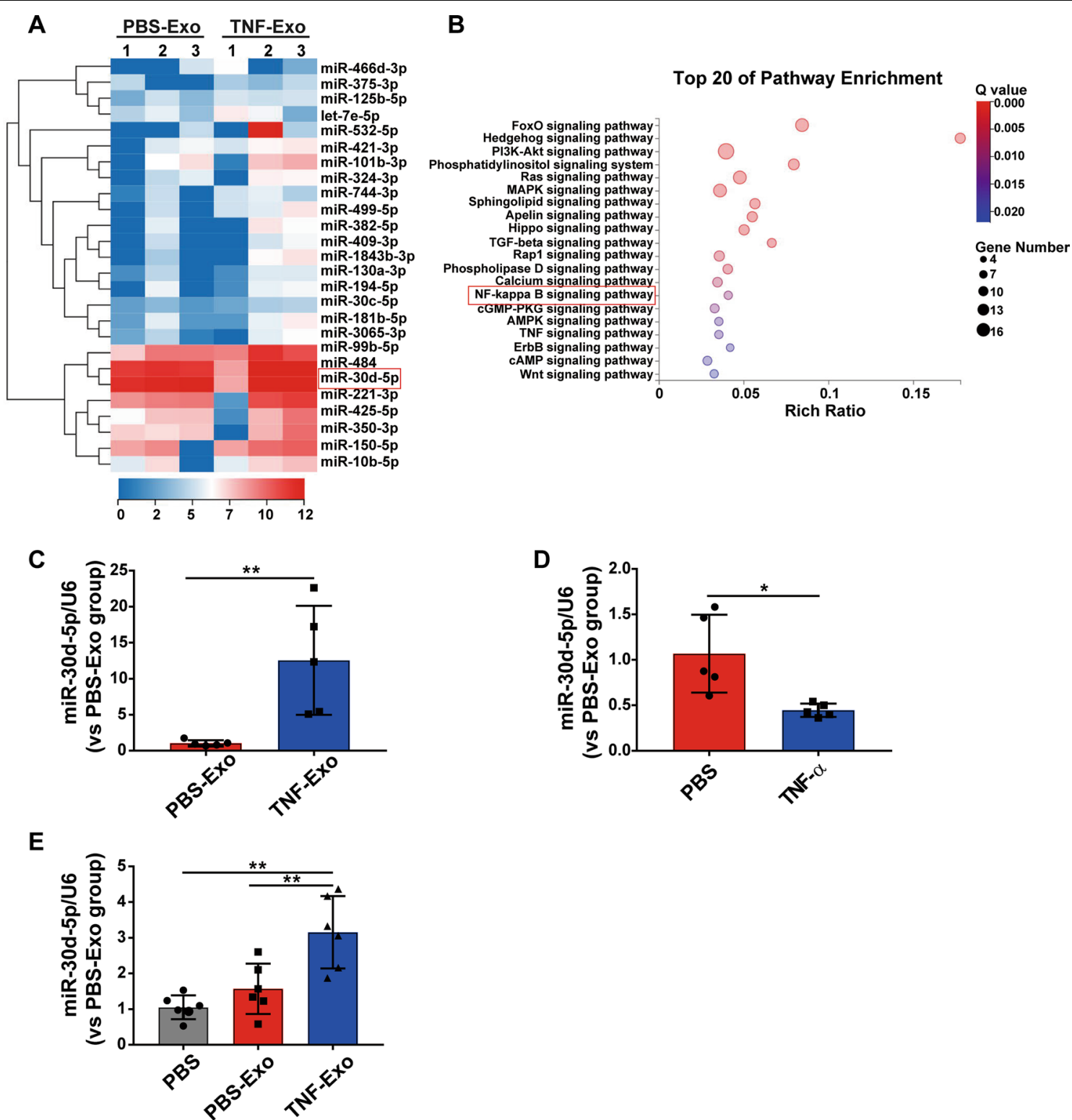

Fig. 3 miRNA analysis of PMN-derived exosomes. a Heat map of exosomal miRNA-seq $(n=3)$. The fluorescence intensity of 26 differentially expressed miRNAs ( $\geq$ twofold) is illustrated from high (red) to low (blue). b Kyoto Encyclopedia of Genes and Genomes (KEGG) analysis on differentially expressed exosomal miRNAs; the 20 most enriched pathways related to signaling transduction are shown. The rich ratio indicates the number of genes in the miRNA target list over the total genes in the respective canonical pathway. c-e Expression of miR-30d-5p in PMN-derived exosomes, in PMNs stimulated with PBS/TNF- $a$ and in recipient Raw264.7 macrophages treated with PBS/PBS-Exo/TNF-Exo by RT-qPCR. Student's $t$ test (c, d) or one-way analysis of variance with Tukey's multiple comparisons test (E) was used for the analysis. Graphs represent means \pm SEM, $n \geq 3$; ${ }^{*} P<0.05,{ }^{* *} P<0.01$ compared within two groups

conducted a dual luciferase reporter assay and found that luciferase activity was markedly reduced by miR30d-5p overexpression in SOCS-1/SIRT1 3'-UTR WT group, but not in $3^{\prime}$-UTR Mut group (Fig. 5b). In Raw264.7 macrophages, overexpression of miR30d-5p suppressed both the mRNA and protein levels of SOCS-1 and SIRT1 (Fig. $5 \mathrm{c}-\mathrm{d}$ ). All these results demonstrate that SOCS-1 and SIRT1 were the direct target genes of miR-30d-5p.

To determine whether exosomal miR-30d-5p targeted SOCS-1/SIRT1 in macrophages, we examined the expression levels of SOCS-1 and SIRT1 and found that both mRNA and protein expressions of SOCS-1 and SIRT1 were decreased in TNF-Exo-treated macrophages 
(Fig. 5e-f), while miR-30d-5p inhibitors reversed the expression of SOCS-1 and SIRT1 (Fig. 5g). In addition, previous studies indicated that SIRT1 reduced NF- $\mathrm{kB}$ activity by decreasing the acetylation level of lysine 310 of the NF- $\mathrm{kB}$ p65 subunit [21]. Indeed, the enhancement of p65 lysine 310 acetylation after TNF-Exo was observed in our study (Fig. 5f), which decreased after miR-30d-5p inhibition (Fig. 5g). These results suggest that exosomal miR-30d-5p targeted SOCS-1 and SIRT1 in macrophages and subsequently activated NF- $\mathrm{kB}$ partly by increasing acetylation of lysine 310 of $\mathrm{p} 65$.

\section{miR-30d-5p inhibition alleviates TNF-Exo or CLP-induced lung injury}

We next investigated the functional role of miR-30d-5p in TNF-Exo in vivo. It was found that miR-30d-5p expression was significantly increased in the lung tissue after TNF-Exo i.p. injection (Fig. 6a). Next, miR-30d-5p inhibitors or scrambled negative control were administered via the tail vein of mice before TNF-Exo injection, and the expression levels of proinflammatory cytokines and NLRP3 inflammasomes in the lung tissues were tested. The results showed that miR-30d-5p inhibitors significantly decreased the levels of IL-1 $\beta$, iNOS, NLRP3, caspase-1 mRNA expressions following TNF-Exo administration (Fig. $6 \mathrm{~b}-\mathrm{c}$ ). In addition, inhibition of miR30d-5p decreased TNF-Exo-induced M1 macrophage activation and macrophage death in the lung (Fig. 6d, e), as well as the histological lesions (Fig. 6f).

In addition, the CLP mouse model was applied to mimic sepsis to further confirm the roles of miR-30d-5p in vivo. Likewise, miR-30d-5p expression was also significantly increased in the lung tissues of CLP mice (Fig. 6g). The decline in IL-6, iNOS, NLRP3 mRNA expression (Fig. 6h, i), the decrease in M1 macrophage activation and macrophage death in the lung (Fig. 6j, k), and the alleviation of lung injury were also observed in the CLP model with miR-30d-5p inhibition (Fig. 6l). Notably, the survival rate in CLP mice with miR-30d-5p inhibition was significantly higher than that without inhibition (Fig. 6m). All these data demonstrated that inhibition of miR-30d-5p could improve both TNF-Exo and CLP-induced lung injury through suppressing M1 macrophage activation and macrophage death.

\section{Discussion}

It was found in our study that exosomal miR-30d-5p from PMNs induced macrophage M1 polarization and primed macrophages pyroptosis by activating NF-kB signaling via targeting SOCS-1 and SIRT1. In addition, we discovered a previously unidentified role of $\mathrm{PMN}-\mathrm{M} \phi$ interaction in promoting inflammation in sepsis-related ALI.

In the early stage of sepsis, neutrophils are thought to be the primary innate immune cells that causing damage to host tissues [31]. In addition to releasing important cytokines, chemokines, ROS and NETs, some studies suggested that PMN-derived exosomes were a new subcellular entity, working as a fundamental link between PMN-driven inflammation and tissue damage [8]. TNF- $\alpha$ plays a central role in the pathogenesis of sepsis and is an early regulator of the immune response [32]. Previous studies $[33,34]$ showed that TNF- $\alpha$ and IL-1 $\beta$ produced by macrophages activated neutrophils during sepsis, and high concentrations of TNF- $\alpha$ and IL-1 $\beta$ have been reported in BALF from ARDS patients. Therefore, in this study, we used TNF- $\alpha$ to activate PMNs from healthy mice and isolated exosomes from the supernatant.

Macrophages have been shown to be the recipient cells for exogenous exosomes and in direct contact with peripheral serum exosomes [35]. A recent study showed that peripheral serum exosomes promoted M1 macrophage polarization and inflammation during sepsis-related ALI, but the study did not address the cellular origin of the circulating exosomes [36]. Our results showed that exosomes isolated from the supernatant of PMNs stimulated with TNF- $\alpha$ promoted M1 macrophage activation both in vivo and in vitro. We also observed that TNF-Exo resulted in a significant lung inflammatory response, suggesting that exosomes released from TNF- $\alpha$-activated PMNs are a kind of pro-inflammatory exosomes and play an important role in sepsis-related ALI.

In addition, we observed that ATP/nigericin significantly upregulated pyroptotic cell death in TNF-Exoprimed macrophages. Induction of pyroptotic cell death in vitro usually needs two signals: the priming signal and the secondary signal. The priming signal upregulates NLRP3 inflammasome and pro-IL-1 $\beta$ expression levels through the transcription factor NF-kB. After

(See figure on next page.)

Fig. 4 TNF-Exo activates NF-KB signaling pathway in macrophage via miR-30d-5p. a-e Prior to co-culturing with TNF-Exo for 24 h, Raw264.7 macrophages were transfected with negative control (NC) or miR-30d-5p inhibitors (anti-miR-30d-5p) for $24 \mathrm{~h}$. $\mathbf{a}, \mathbf{b}$ Flow cytometry detection of CD11c and CD86 expression. c Detection of inflammatory cytokine mRNA (IL-6, IL-1 $\beta$, TNF-a) and iNOS mRNA expression by RT-qPCR. $\mathbf{d}$ Western blot of NF-KB p-p65 and p65 in Raw264.7 macrophages. e Detection of NLRP3 and caspase-1 mRNA expression by RT-qPCR. f-h Transfection of Raw264.7 macrophages with negative control (NC) or miR-30d-5p inhibitors for $24 \mathrm{~h}$, followed by stimulation with TNF-Exo plus ATP. f Flow cytometry detection of Alexa Fluor 488-labeled caspase-1 FLICA expression. g Western blots of pro-caspase-1 (Pro-casp-1) and activated/cleaved caspase-1 (Casp-1 p20) in whole-cell lysates of Raw264.7. h Cleavage of GSDMD by immunoblotting. Student's t test was used for analysis. Graphs represent means $\pm S E M, n \geq 3 ;{ }^{*} P<0.05,{ }^{*} P<0.01$ compared within two groups 

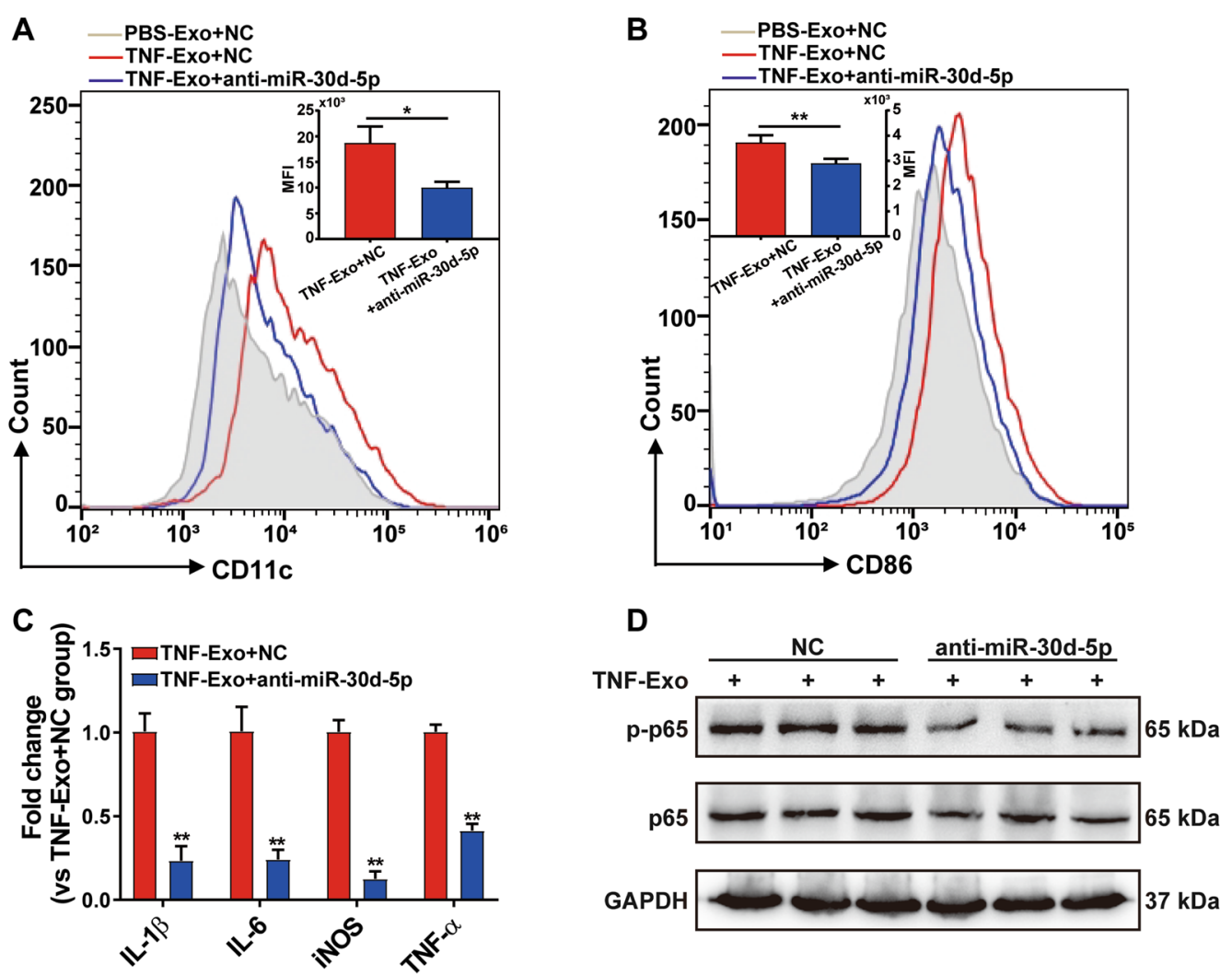

E
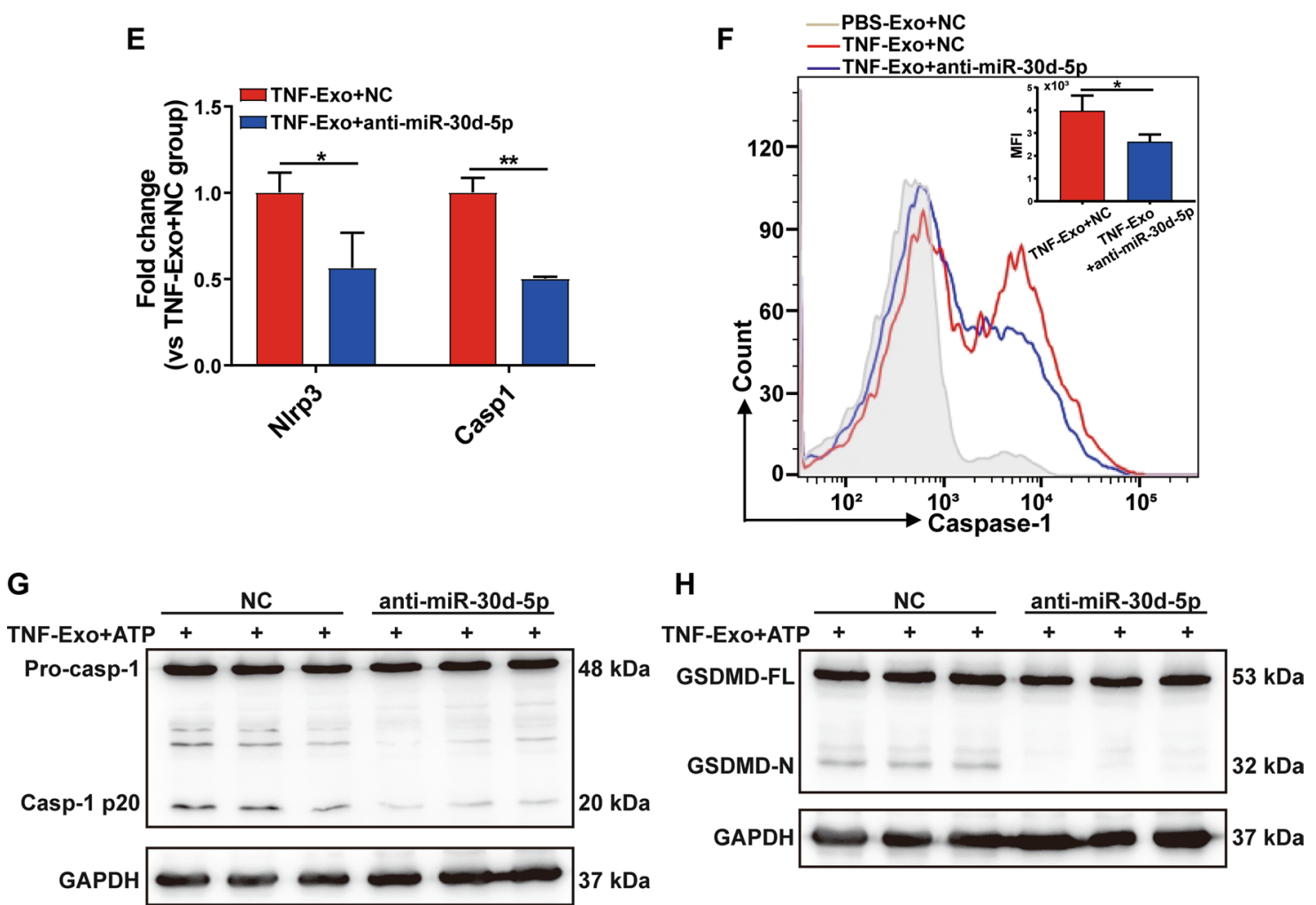

Fig. 4 (See legend on previous page.) 


\section{A SOCS-1 3'UTR Mut 5'...CUCCUACCUCUCCAgGaUgagau... 3' SOCS-1 3'UTR WT 5'....CUCCUACCUCUCCAUGUUUACA U....3' mmu-miR-30d-5p 3'....GAAGGUCAGCCCCUACAAAUGU...5' SIRT1 3'UTR WT 5'...UGUCAAAAAAUGAAUGUUUACU...3' SIRT1 3'UTR Mut 5'....UGUCAAAAAAUGAAGGAUGAGU...3'}

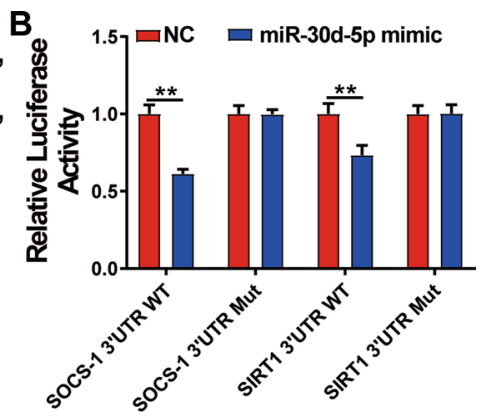

C

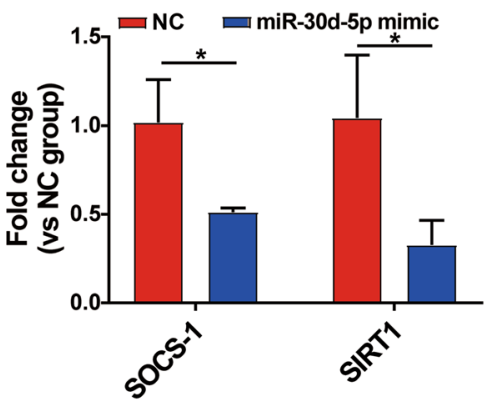

D
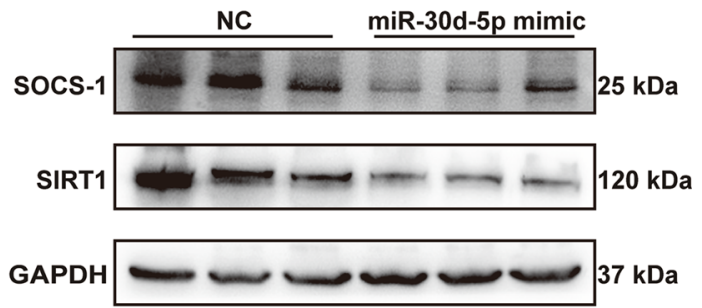

E

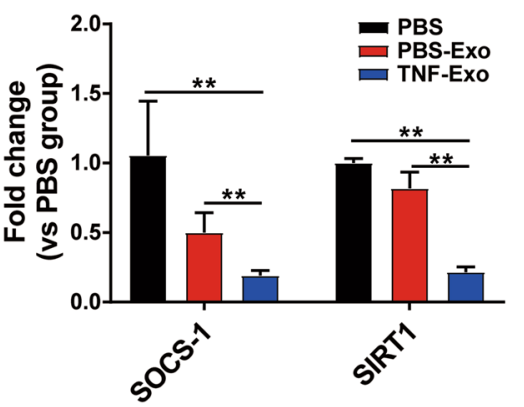

$\mathbf{F}$

PBS PBS-Exo INF-Exo socs-1

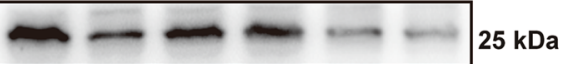

SIRT1 $--\cdots$ $120 \mathrm{kDa}$ p65-Acetyl310 $--\infty-\infty-\infty 65 \mathrm{kDa}$
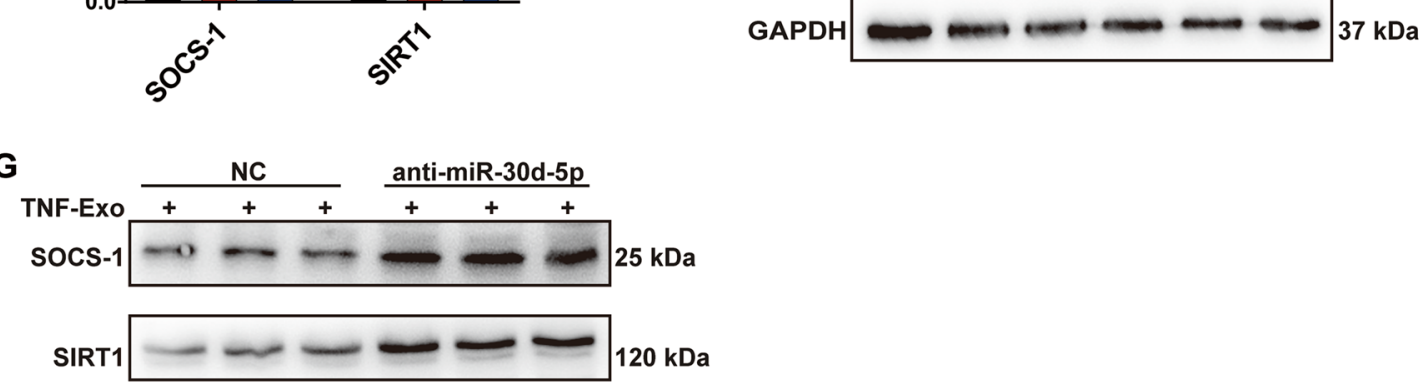

p65-Acetyl310

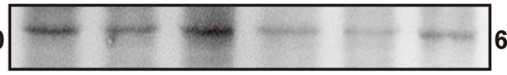

$65 \mathrm{kDa}$

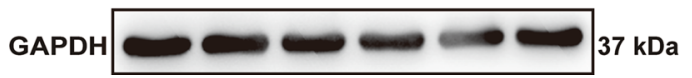

Fig. 5 Exosomal miR-30d-5p activates NF-KB in macrophage via targeting SOCS-1 and SIRT1. a Sequence alignment between miR-30d-5p and its putative binding sites (in red letters) in the SOCS-1/SIRT1 3'-UTR. Mutation of the miR-30d-5p target sites (in blue letters) is also shown. b Detection of the relative luciferase activities of WT and Mut SOCS-1/SIRT1 reporters by luciferase reporter assay, using Renilla luciferase vector as the internal control. RT-qPCR analysis of relative SOCS-1/SIRT1 mRNA level (c) and Western blot (d) of SOCS-1 and SIRT1 in Raw264.7 macrophages transfected with miR-30d-5p mimics as indicated. e, f Treatment of Raw264.7 macrophages with PBS/PBS-Exo/TNF-Exo for $24 \mathrm{~h}$. e Detection of mRNA levels of SOCS-1 and SIRT1 by RT-qPCR. f Western blot analysis of SOCS-1, SIRT1 and p65-Acetyl 310. g Prior to co-culturing with TNF-Exo for 24 h, Raw264.7 macrophages were transfected with control or miR-30d-5p inhibitors for $24 \mathrm{~h}$. The expression levels of SOCS-1, SIRT1 and p65-Acetyl 310 in Raw264.7 cells were measured by Western blot. Student's t test (b, c) or one-way analysis of variance with Tukey's multiple comparisons test (e) was used for the analysis. Graphs represent means \pm SEM, $n \geq 3 ;{ }^{*} P<0.05$, ${ }^{*} P<0.01$ compared within two groups 


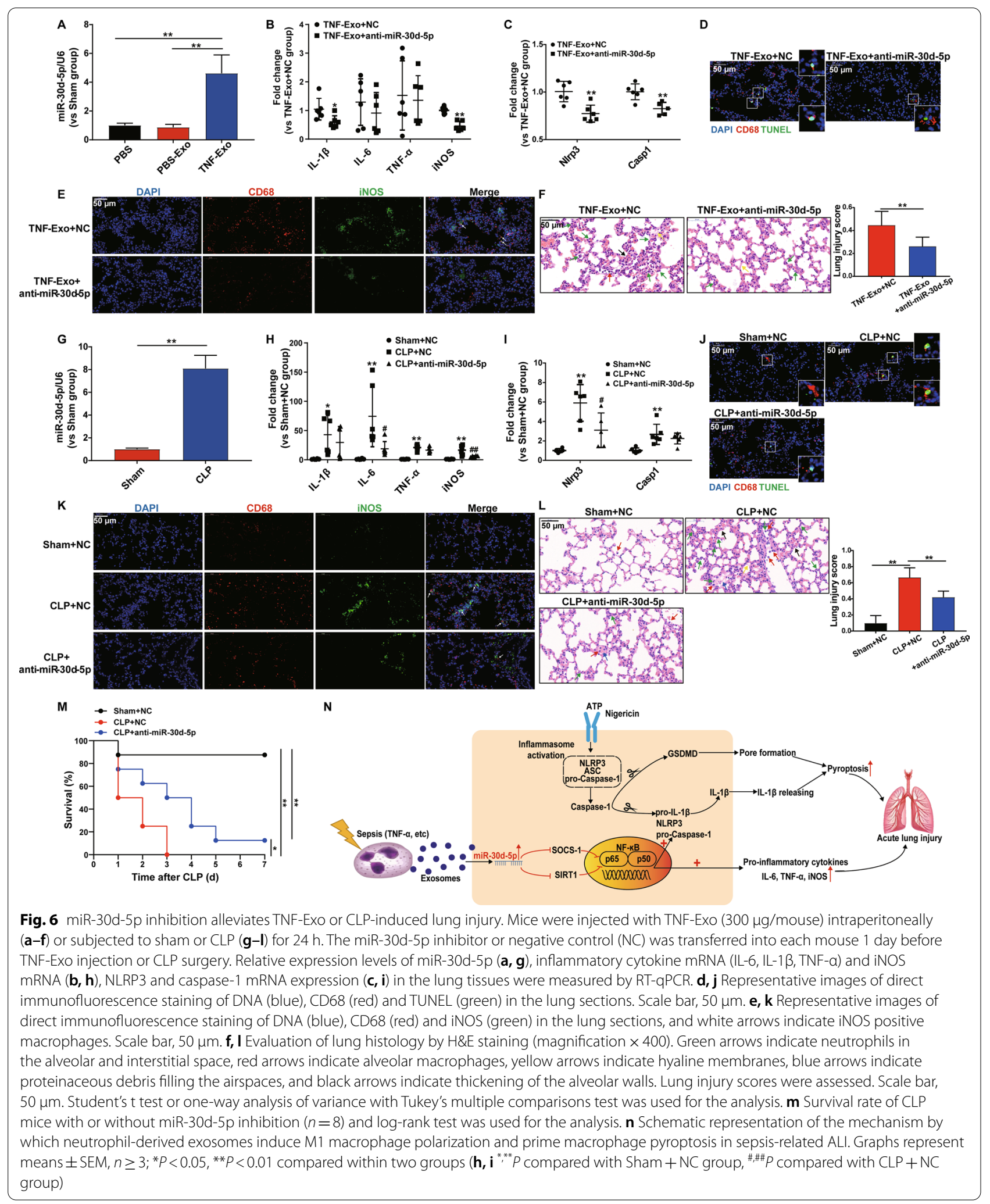


the priming phase, the secondary signal, such as ATP/ nigericin, initiates the assembly of several protein complexes, including NLRP3, apoptosis-associated specklike protein containing CARD (ASC) and pro-caspase-1, by regulating the formation of the ASC pyroptosome and splicing of caspase-1 into its active form [27]. Based on our results, TNF-Exo served as a priming signal to increase NLRP3 inflammasome expression through activating NF- $\mathrm{kB}$ signaling pathway, which still required the secondary signal to finally induce pyroptotic cell death. High concentrations of extracellular ATP have been implicated in multiple in vivo inflammatory responses, including lung inflammation and fibrosis, systemic inflammation and tissue damage during endotoxemia [37, 38]. Almost all mammalian cells, including myeloid cells, platelets, leukocytes, epithelial and endothelial cells, can release ATP in response to stimulation [39], which may explain why TNF-Exo could promote macrophage pyroptosis in vivo, as TNF-Exo or cytokines upregulated by TNF-Exo may stimulate other cells to release high concentrations of ATP. However, the exact mechanisms need to be further addressed.

Exosomally transferred miRNAs have emerged as novel regulators of cellular function. miRNA sequencing and literature search in our study suggest that miR-30d-5p may be the functional molecule within TNF-Exo. Our study here reported a novel function of miR-30d-5p in exosomes as a regulator of macrophage polarization and pyroptosis. The exosome-mediated inflammatory pathway may be a new mechanism responsible for the development of sepsis-related ALI by promoting PMN-M $\phi$ communication.

Finally, we further demonstrated the role of miR30d-5p in TNF-Exo and CLP-induced lung injury. The expression level of miR-30d-5p was significantly increased in the lung after TNF-Exo administration, suggesting that miR-30d-5p could be transferred to the lung tissue via exosomes. miR-30d-5p loss-of-function markedly reduced M1 macrophage activation and death in the lung, and ameliorated lung injury, indicating that miR30d-5p contributed to TNF-Exo-induced lung injury. Furthermore, inhibition of miR-30d-5p was found to be highly related to the improvement of lung injury and survival rate in the experimental sepsis model, which may provide a novel molecular target for the treatment of sepsis-related ALI.

There were several limitations in our study. Firstly, more studies about the correlations of miR-30d-5p with clinical parameters such as oxygenation index and mortality of sepsis-related ARDS patients are required to make our conclusions more informative and reliable. Secondly, we found that TNF-Exo in vivo could promote macrophage pyroptosis and TNF-Exo in vitro needed a second signal to finally induce macrophage pyroptosis, based on which raised the hypothesis that TNF-Exo or cytokines increased by TNF-Exo may stimulate other cells to release high concentrations of ATP in vivo, which served as a second signal to induce TNF-Exo-primed macrophage pyroptosis. However, the exact mechanisms need to be further addressed. Lastly, although specially inhibition of miR-30d-5p in PMNs in vivo is not easily manipulated, administration of imR-30d-5p inhibitors via the tail vein of mice before CLP in our study did exhibit a protective effect on lung injury and survival, suggesting that miR-30d-5p may represent a new therapeutic target for the progression of sepsis-related ALI.

\section{Conclusions}

The data obtained in our study demonstrated that exosomal miR-30d-5p derived from PMNs could promote lung inflammation by enhancing M1 macrophage polarization and priming macrophage pyroptosis. Modulating the cross-talk between PMNs and macrophages attenuated tissue inflammation and injury during sepsis-related ALI, highlighting its potential as a therapeutic strategy in sepsis-related ARDS.

\begin{abstract}
Abbreviations
PMNs: Polymorphonuclear neutrophils; ALI: Acute lung injury; TNF-a: Tumor necrosis factor-a; $M \varphi$ : Macrophage; BMDMs: Bone marrow-derived macrophages; NLRP3: NOD-like receptor 3; NF-kB: Nuclear factor kB; SOCS-1: Suppressor of cytokine signaling; SIRT1: Sirtuin 1; CLP: Cecal ligation and puncture; ARDS: Acute respiratory distress syndrome; miRNAs: MicroRNAs; IL13: Interleukin 13; GSDMD: Gasdermin D; NETs: Neutrophil extracellular traps; WT: Wild type; PLF: Peritoneal lavage fluid; PM $\varphi$ : Peritoneal macrophage; RPMI: Roswell park memorial institute; FBS: Fetal bovine serum; PBS: Phosphate buffer saline; Exos: Exosomes; H\&E: Hematoxylin and eosin; iNOS: Inducible nitric oxide synthase; RT-PCR: Real-time polymerase chain reaction; TEM: Transmission electron microscopy; ASC: Apoptosis-associated speck-like protein containing CARD.
\end{abstract}

\section{Supplementary Information}

The online version contains supplementary material available at https://doi. org/10.1186/s13054-021-03775-3.

Additional file 1. Supplementary methods

Additional file 2 . Supplementary results

Additional file 3. Proteomic data

Acknowledgements

Not applicable.

Authors' contributions

YJ, TZ and CMZ contributed equally to this work. YJ, ZLM and XYS contributed to the conception and design. YJ, TZ, CMZ and HYJ performed the experiments, analyzed data, and wrote the manuscript. XYT and RX participated in the animal experiments. WW helped to revise the manuscript. All authors read and approved the final manuscript. 


\section{Funding}

This work was supported by the National Natural Science Foundation of China (Grant No. 81974292 to X.Y.S, 82102257 to Y. J), and the Key Program of Shanghai Committee of Science and Technology (Grant No. 18411951200 to X.Y.S).

\section{Availability of data and materials}

The datasets generated and analyzed during the current study are available from the corresponding author on reasonable request.

\section{Declarations}

\section{Ethics approval and consent to participate}

All animal experiments were conducted under the rules approved by the Ethics Committee of Xinhua Hospital Affiliated to Shanghai Jiao Tong University School of Medicine (Approval No. XHEC-F-2020-019).

\section{Consent for publication}

All listed authors consent to the submission, and all data are used with the consent of the person generating the data.

\section{Competing interests}

The authors declare that they have no competing interests.

\section{Author details}

'Department of Anesthesiology, Nanjing Drum Tower Hospital, The Affiliated Hospital of Nanjing University Medical School, 321 Zhongshan Road, Nanjing 210008, China. ${ }^{2}$ Department of Anesthesiology and Intensive Care Unit, Xinhua Hospital, School of Medicine, Shanghai Jiaotong University, 1665 Kongjiang Road, Shanghai 200092, China. ${ }^{3}$ National Clinical Research Center of Kidney Diseases, Jinling Hospital, Nanjing University School of Medicine, Nanjing, China.

Received: 27 June 2021 Accepted: 27 September 2021

\section{Published online: 12 October 2021}

\section{References}

1. Fleischmann C, Scherag A, Adhikari N, Hartog C et al (2016) Assessment of global incidence and mortality of hospital-treated sepsis. current estimates and limitations. Am J Respir Crit Care Med 193(3): 259-272

2. Singer M, Deutschman C, Seymour C, Shankar-Hari M, et al. The third international consensus definitions for sepsis and septic shock (sepsis-3). JAMA. 2016:315(8):801-10.

3. Moreira J. Severe sepsis and septic shock. N Engl J Med. 2013:369(21):2063.

4. Liu L, Sun B. Neutrophil pyroptosis: new perspectives on sepsis. Cell Mol Life Sci CMLS. 2019;76(11):2031-42.

5. Thebaud B, Stewart DJ. Exosomes: cell garbage can, therapeutic carrier, or Trojan horse? Circulation. 2012;126(22):2553.

6. Li M, Zeringer E, Barta T, Schageman J, et al. Analysis of the RNA content of the exosomes derived from blood serum and urine and its potential as biomarkers. Philos Trans R Soc B Biol Sci. 2014;369:1652.

7. Vargas A, Roux-Dalvai F, Droit A, Lavoie J. Neutrophil-derived exosomes: a new mechanism contributing to airway smooth muscle remodeling. Am J Respir Cell Mol Biol. 2016;55(3):450-61.

8. Genschmer K, Russell D, Lal C, Szul T, et al. Activated PMN exosomes: pathogenic entities causing matrix destruction and disease in the lung. Cell. 2019;176:113-126.e115.

9. Bazzan $E$, Turato $G$, Tinè $M$, Radu CM, et al. Dual polarization of human alveolar macrophages progressively increases with smoking and COPD severity. Respir Res. 2017;18(1):40.

10. Murray P, Allen J, Biswas S, Fisher E, et al. Macrophage activation and polarization: nomenclature and experimental guidelines. Immunity. 2014;41(1):14-20

11. Galluzzi L, Bravo-San Pedro J, Vitale I, Aaronson S, et al. Essential versus accessory aspects of cell death: recommendations of the NCCD 2015. Cell Death Differ. 2015:22(1):58-73.

12. Bordon Y. Mucosal immunology: inflammasomes induce sepsis following community breakdown. Nat Rev Immunol. 2012;12(6):400-1.
13. Pu Q, Gan C, Li R, Li Y, et al. PseudomonasAtg7 deficiency intensifies inflammasome activation and pyroptosis in sepsis. J Immunol (Baltimore, Md: 1950). 2017:198(8):3205-13.

14. Chan F, Luz N, Moriwaki K. Programmed necrosis in the cross talk of cell death and inflammation. Annu Rev Immunol. 2015:33:79-106.

15. Fan J, Li Y, Vodovotz Y, Billiar T, et al. Neutrophil NAD(P)H oxidase is required for hemorrhagic shock-enhanced TLR2 up-regulation in alveolar macrophages in response to LPS. Shock (Augusta, Ga). 2007:28(2):213-8

16. Fan J, Malik AB. Toll-like receptor-4 (TLR4) signaling augments chemokine-induced neutrophil migration by modulating cell surface expression of chemokine receptors. Nat Med. 2003;9(3):315.

17. Jiao Y, Li Z, Loughran PA, Fan EK, et al. Frontline Science: Macrophagederived exosomes promote neutrophil necroptosis following hemorrhagic shock. J Leukocyte Biol; 2017:jlb.3HI0517-0173R

18. Warnatsch A, loannou M, Wang Q, Papayannopoulos V. Inflammation. Neutrophil extracellular traps license macrophages for cytokine production in atherosclerosis. Science. 2015;349(6245):316-20.

19. Chen L, Zhao Y, Lai D, Zhang P, et al. Neutrophil extracellular traps promote macrophage pyroptosis in sepsis. Cell Death Dis. 2018:9(6):597.

20. Lv L, Feng Y, Wu M, Wang B, et al. Exosomal miRNA-19b-3p of tubular epithelial cells promotes M1 macrophage activation in kidney injury. Cell Death Differ. 2020;27(1):210-26.

21. Shen G, Ren H, Shang Q, Zhang Z, et al. miR-128 plays a critical role in murine osteoclastogenesis and estrogen deficiency-induced bone loss. Theranostics. 2020;10(10):4334-48.

22. Xu F, Zhang C, Zou Z, Fan E, et al. Aging-related Atg 5 defect impairs neutrophil extracellular traps formation. Immunology. 2017:151(4):417-32.

23. Matute-Bello G, Downey G, Moore B, Groshong S, et al. An official American Thoracic Society workshop report: features and measurements of experimental acute lung injury in animals. Am J Respir Cell Mol Biol. 2011:44(5):725-38

24. Keshari R, Jyoti A, Dubey M, Kothari N, et al. Cytokines induced neutrophil extracellular traps formation: implication for the inflammatory disease condition. PLoS ONE. 2012;7(10):e48111.

25. Miyazaki Y, Inoue T, Kyi M, Sawada M, et al. Effects of a neutrophil elastase inhibitor (ONO-5046) on acute pulmonary injury induced by tumor necrosis factor alpha (TNFalpha) and activated neutrophils in isolated perfused rabbit lungs. Am J Respir Crit Care Med. 1998;157(1):89-94.

26. Okeke E, Louttit C, Fry C, Najafabadi A, et al. Inhibition of neutrophil elastase prevents neutrophil extracellular trap formation and rescues mice from endotoxic shock. Biomaterials. 2020;238:119836.

27. Xue Z, Xi Q, Liu H, Guo X, et al. miR-21 promotes NLRP3 inflammasome activation to mediate pyroptosis and endotoxic shock. Cell Death Dis. 2019;10(6):461

28. Bauernfeind F, Horvath G, Stutz A, Alnemri E, et al. Cutting edge: NF-kappaB activating pattern recognition and cytokine receptors license NLRP3 inflammasome activation by regulating NLRP3 expression. J Immunol (Baltimore, Md: 1950). 2009;183(2):787-91.

29. Real J, Ferreira L, Esteves G, Koyama F, et al. Exosomes from patients with septic shock convey miRNAs related to inflammation and cell cycle regulation: new signaling pathways in sepsis? Critical care (London, England). 2018;22(1):68

30. Han $X$, Zhang L, Liu Y, Wu M, et al. Resveratrol protects $H 9 c 2$ cells against hypoxia-induced apoptosis through miR-30d-5p/SIRT1/NF-kB axis. J Biosci. 2020;45:42.

31. Kolaczkowska E, Kubes P. Neutrophil recruitment and function in health and inflammation. Nat Rev Immunol. 2013;13(3):159-75.

32. Leon L, White A, Kluger M. Role of IL-6 and TNF in thermoregulation and survival during sepsis in mice. Am J Physiol. 1998;275(1):R269-277.

33. Moldoveanu B, Otmishi P, Jani P, Walker J, et al. Inflammatory mechanisms in the lung. J Inflamm Res. 2009;2:1-11.

34. Park WY, Goodman RB, Steinberg KP, Ruzinski JT, et al. Cytokine balance in the lungs of patients with acute respiratory distress syndrome. Am J Respir Crit Care Med. 2001;164(10 Pt 1):1896.

35. Wu L, Zhang $X$, Zhang B, Shi H, et al. Exosomes derived from gastric cancer cells activate NF-kB pathway in macrophages to promote cancer progression. Tumour Biol. 2016;37(9):12169-80. 
36. Jiang K, Yang J, Guo S, Zhao G, et al. Peripheral Circulating exosomemediated delivery of miR-155 as a novel mechanism for acute lung inflammation. Mol Ther J Am Soc Gene Therapy. 2019;27(10):1758-71.

37. Cauwels $R$, Vandendriessche $S$, et al. Extracellular ATP drives systemic inflammation, tissue damage and mortality. Cell Death Dis. 2014;5:e1 102.

38. Extracellular ATP. is a danger signal activating P2X7 receptor in lung inflammation and fibrosis. Am J Respir Crit Care Med. 2010;182(6):774-83.
39. Junger WG. Immune cell regulation by autocrine purinergic signalling. Nat Rev Immunol. 2011;11(3):201-12.

\section{Publisher's Note}

Springer Nature remains neutral with regard to jurisdictional claims in published maps and institutional affiliations.
Ready to submit your research? Choose BMC and benefit from:

- fast, convenient online submission

- thorough peer review by experienced researchers in your field

- rapid publication on acceptance

- support for research data, including large and complex data types

- gold Open Access which fosters wider collaboration and increased citations

- maximum visibility for your research: over 100M website views per year

At BMC, research is always in progress.

Learn more biomedcentral.com/submissions 